\title{
The 25th and 26th Mersenne Primes
}

\author{
By Curt Noll and Laura Nickel
}

Abstract. The 25th and 26th Mersenne primes are $2^{21701}-1$ and $2^{23209}-1$, respectively. Their primality was determined with an implementation of the LucasLehmer test on a CDC Cyber 174 computer. The 25 th and 26 th even perfect numbers are $\left(2^{21701}-1\right) 2^{21700}$ and $\left(2^{23209}-1\right) 2^{23208}$, respectively.

Definitions.

Mersenne number - an integer of the form $M_{n}=2^{n}-1$.

Mersenne prime - a Mersenne number that is prime.

Perfect number - a natural number $n$ such that the factors of $n$ less than $n$ sum to $n$.

It is known that if $M_{p}$ is prime, $p$ is prime; the converse is false. The test for primality was discovered by Lucas in 1876 and improved upon by Lehmer in 1930. The test, known as the Lucas-Lehmer test, is as follows:

Given an odd prime $p$

let $u_{2}=4$,

$$
\begin{aligned}
& u_{3} \equiv u_{2}^{2}-2\left(\bmod 2^{p}-1\right) \\
& \vdots \\
& u_{n} \equiv \dot{u}_{n-1}^{2}-2\left(\bmod 2^{p}-1\right),
\end{aligned}
$$

then $2^{p}-1$ is prime iff $u_{p} \equiv 0[3]$.

To implement the test, we represented the required numbers in $d=\lceil p / 47\rceil$ digits in base $2^{47}=\beta$. The squaring of the multi-precision integers,

$$
u=\sum_{i=1}^{d-1} X_{i} \beta^{i}
$$

was performed in the following manner:

$$
u^{2}=\sum_{k=0}^{d-1} X_{k}^{2} \beta^{2 k}+\sum_{k=1}^{2 d-3} \beta^{k} \cdot 2 \sum X_{i} X_{j}
$$

(where the rightmost summation runs over all $(i, j)$ such that $i+j=k$ and $0 \leqslant i<$ $j \leqslant d-1$ ). Because the Cyber 174 floating multiply unit operates on integers in a special manner we were able to use the floating and integer multiply instructions to achieve the required 94 bit cross products.

In 1963 Gillies announced that $M_{9689}, M_{9941}$, and $M_{11213}$ were prime after testing the range from $M_{5000}$ to $M_{11400}$ [1]. In 1971, Tuckerman announced that 
$M_{19937}$ was prime after testing through $M_{20000}$ [8]. We later learned that Tuckerman continued testing, and stopped at $M_{21000}$ without finding another prime [9].

We first eliminated $31 M_{p}$ for $21000<p<21701$ by using a factor table prepared by Wagstaff [10]. In this table, Wagstaff gave factors $<2^{35}$ for $M_{p}$. On October 30,1978 at 9:40 pm, we found $M_{21701}$ to be prime [6]. The CPU time required for this test was 7:40:20. Tuckerman and Lehmer later provided confirmation of this result [4], [9].

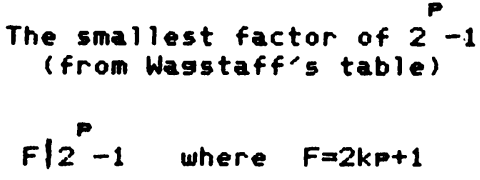

\begin{tabular}{|c|c|c|c|c|c|c|c|}
\hline$P$ & $k$ & $p$ & $k$ & $P$ & $k$ & $p$ & $k$ \\
\hline $\begin{array}{l}21011 \\
21019 \\
21023 \\
21061 \\
21067\end{array}$ & $\begin{array}{r}1 \\
320 \\
25 \\
8 \\
9\end{array}$ & $\begin{array}{l}21997 \\
22031 \\
22039 \\
22063 \\
22073\end{array}$ & $\begin{array}{r}16175 \\
7360 \\
27704 \\
41 \\
3\end{array}$ & $\begin{array}{l}22853 \\
22901 \\
22907 \\
22937 \\
22943\end{array}$ & $\begin{array}{r}3 \\
3831 \\
147604 \\
4 \\
1\end{array}$ & $\begin{array}{l}23629 \\
23663 \\
23671 \\
23747 \\
23761\end{array}$ & $\begin{array}{r}1047 \\
220 \\
708 \\
4 \\
33095\end{array}$ \\
\hline $\begin{array}{l}21089 \\
21107 \\
21121\end{array}$ & $\begin{array}{r}172 \\
60469 \\
5520\end{array}$ & $\begin{array}{l}22079 \\
22093 \\
22109\end{array}$ & $\begin{array}{r}1 \\
190835 \\
432\end{array}$ & $\begin{array}{l}22963 \\
22993 \\
23003\end{array}$ & $\begin{array}{r}12 \\
3 \\
25\end{array}$ & $\begin{array}{l}23801 \\
23819 \\
23831\end{array}$ & $\begin{array}{r}120 \\
13\end{array}$ \\
\hline $\begin{array}{l}21179 \\
21221 \\
21227\end{array}$ & $\begin{array}{r}1 \\
15 \\
4\end{array}$ & $\begin{array}{l}22123 \\
22147 \\
22157\end{array}$ & $\begin{array}{r}53 \\
5 \\
267\end{array}$ & $\begin{array}{l}23017 \\
23021 \\
23027\end{array}$ & $\begin{array}{r}135 \\
7080 \\
185140\end{array}$ & $\begin{array}{l}23833 \\
23857 \\
23873\end{array}$ & $\begin{array}{r}291 \\
200 \\
3\end{array}$ \\
\hline $\begin{array}{l}21247 \\
21313 \\
21347\end{array}$ & $\begin{array}{r}44 \\
1595 \\
4\end{array}$ & $\begin{array}{l}22159 \\
22171 \\
22193\end{array}$ & $\begin{array}{r}3260 \\
343605 \\
33396\end{array}$ & $\begin{array}{l}23029 \\
23041 \\
23053\end{array}$ & $\begin{array}{r}267 \\
164 \\
3\end{array}$ & $\begin{array}{l}23879 \\
23887 \\
23917\end{array}$ & $\begin{array}{r}4 \\
36237 \\
3\end{array}$ \\
\hline $\begin{array}{l}21379 \\
21383 \\
21391\end{array}$ & $\begin{array}{r}57 \\
1 \\
272828\end{array}$ & $\begin{array}{l}22247 \\
22259 \\
22271\end{array}$ & $\begin{array}{r}25 \\
1 \\
1\end{array}$ & $\begin{array}{l}23057 \\
23063 \\
23081\end{array}$ & $\begin{array}{l}64 \\
21 \\
51\end{array}$ & $\begin{array}{l}23957 \\
23977 \\
23993\end{array}$ & $\begin{array}{r}3 \\
131355 \\
95551\end{array}$ \\
\hline $\begin{array}{l}21397 \\
21401 \\
21407\end{array}$ & $\begin{array}{r}284 \\
348288 \\
1252\end{array}$ & $\begin{array}{l}22273 \\
22277 \\
22279\end{array}$ & $\begin{array}{r}105800 \\
2427 \\
1785\end{array}$ & $\begin{array}{l}23087 \\
23099 \\
23131\end{array}$ & $\begin{array}{r}12 \\
1 \\
473\end{array}$ & $\begin{array}{l}24007 \\
24019 \\
24023\end{array}$ & $\begin{array}{r}80 \\
276 \\
12\end{array}$ \\
\hline $\begin{array}{l}21419 \\
21433 \\
21493\end{array}$ & $\begin{array}{l}1 \\
3 \\
3\end{array}$ & $\begin{array}{l}22291 \\
22343 \\
22349\end{array}$ & $\begin{array}{r}3041 \\
1 \\
7\end{array}$ & $\begin{array}{l}23143 \\
23167 \\
23197\end{array}$ & $\begin{array}{r}16868 \\
2849 \\
27\end{array}$ & $\begin{array}{l}24029 \\
24043 \\
24049\end{array}$ & $\begin{array}{r}267 \\
1320 \\
10475\end{array}$ \\
\hline $\begin{array}{l}21521 \\
21529 \\
21557\end{array}$ & $\begin{array}{r}4 \\
560 \\
661587\end{array}$ & $\begin{array}{l}22369 \\
22381 \\
22397\end{array}$ & $\begin{array}{r}95 \\
191 \\
255\end{array}$ & $\begin{array}{l}23251 \\
23269 \\
23279\end{array}$ & $\begin{array}{r}36 \\
60 \\
1\end{array}$ & $\begin{array}{l}24061 \\
24097 \\
24103\end{array}$ & $\begin{array}{l}15219 \\
54960 \\
34997\end{array}$ \\
\hline $\begin{array}{l}21587 \\
21611 \\
21617\end{array}$ & $\begin{array}{r}9 \\
1 \\
427\end{array}$ & $\begin{array}{l}22409 \\
22433 \\
22447\end{array}$ & $\begin{array}{r}15 \\
541803 \\
8\end{array}$ & $\begin{array}{l}23293 \\
23297 \\
23311\end{array}$ & $\begin{array}{r}8 \\
4 \\
9645\end{array}$ & $\begin{array}{l}24107 \\
24109 \\
24137\end{array}$ & $\begin{array}{r}324 \\
27356 \\
7\end{array}$ \\
\hline $\begin{array}{l}21649 \\
21661 \\
21727\end{array}$ & $\begin{array}{r}15 \\
56 \\
180\end{array}$ & $\begin{array}{l}22453 \\
22481 \\
22483\end{array}$ & $\begin{array}{r}11 \\
4 \\
113676\end{array}$ & $\begin{array}{l}23321 \\
23327 \\
23333\end{array}$ & $\begin{array}{r}528 \\
536973 \\
148\end{array}$ & $\begin{array}{l}24169 \\
24179 \\
24203\end{array}$ & $\begin{array}{r}22287 \\
4 \\
1\end{array}$ \\
\hline $\begin{array}{l}21767 \\
21787 \\
21803\end{array}$ & $\begin{array}{r}4 \\
3444 \\
1\end{array}$ & $\begin{array}{l}22511 \\
22531 \\
22543\end{array}$ & $\begin{array}{r}21 \\
6929 \\
4220\end{array}$ & $\begin{array}{l}23339 \\
23369 \\
23371\end{array}$ & $\begin{array}{r}1 \\
11472 \\
116\end{array}$ & $\begin{array}{l}24229 \\
24239 \\
24247\end{array}$ & $\begin{array}{r}360 \\
1 \\
22424\end{array}$ \\
\hline $\begin{array}{l}21817 \\
21821 \\
21839\end{array}$ & $\begin{array}{r}599787 \\
280 \\
1872\end{array}$ & $\begin{array}{l}22549 \\
22567 \\
22619\end{array}$ & $\begin{array}{r}1259 \\
13608 \\
69\end{array}$ & $\begin{array}{l}23399 \\
23417 \\
23459\end{array}$ & $\begin{array}{r}1705 \\
7 \\
1\end{array}$ & $\begin{array}{l}24317 \\
24329 \\
24337\end{array}$ & $\begin{array}{r}1464 \\
36 \\
3\end{array}$ \\
\hline $\begin{array}{l}21841 \\
21863 \\
21871\end{array}$ & $\begin{array}{r}5003 \\
45 \\
993\end{array}$ & $\begin{array}{l}22679 \\
22709 \\
22717\end{array}$ & $\begin{array}{r}9009 \\
19 \\
3\end{array}$ & $\begin{array}{l}23497 \\
23531 \\
23539\end{array}$ & $\begin{array}{r}3 \\
205 \\
365\end{array}$ & $\begin{array}{l}24359 \\
24371 \\
24373\end{array}$ & $\begin{array}{r}16 \\
24 \\
431087\end{array}$ \\
\hline $\begin{array}{l}21893 \\
21929 \\
21937\end{array}$ & $\begin{array}{r}7207 \\
118371 \\
163820\end{array}$ & $\begin{array}{l}22727 \\
22739 \\
22751\end{array}$ & $\begin{array}{r}27117 \\
4 \\
1\end{array}$ & $\begin{array}{l}23557 \\
23567 \\
23593\end{array}$ & $\begin{array}{r}73544 \\
16264 \\
132\end{array}$ & $\begin{array}{l}24379 \\
24391 \\
24413\end{array}$ & $\begin{array}{r}24 \\
5 \\
193552\end{array}$ \\
\hline $\begin{array}{l}21943 \\
21961\end{array}$ & $\begin{array}{r}94436 \\
228\end{array}$ & $\begin{array}{l}22769 \\
22777\end{array}$ & $\begin{array}{r}171564 \\
24\end{array}$ & $\begin{array}{l}23599 \\
23603\end{array}$ & $\begin{array}{r}10700 \\
1\end{array}$ & $\begin{array}{l}24419 \\
24439\end{array}$ & $\begin{array}{r}12 \\
5741\end{array}$ \\
\hline 21977 & 67 & 22807 & 89 & 23609 & 410431 & 24499 & 9 \\
\hline
\end{tabular}


LUCAS RESIDUES (R) FOR. $2^{P}-1$

15

$(\operatorname{MOD} 2)$

\begin{tabular}{cc}
$P$ & $R$ \\
\hline 21001 & 22167 \\
21013 & 43631 \\
21017 & 15476 \\
21031 & 52200 \\
21059 & 54560 \\
21101 & 72022 \\
21139 & 14444 \\
21143 & 17334 \\
21149 & 34525 \\
21157 & 16203 \\
21163 & 54514 \\
21169 & 41315 \\
21187 & 24217 \\
21191 & 57116 \\
21193 & 67705 \\
21211 & 56211 \\
21269 & 32636 \\
21277 & 66175 \\
21283 & 03020 \\
21317 & 66051 \\
21319 & 03332 \\
21323 & 27410 \\
21341 & 44535 \\
21377 & 74317 \\
21467 & 25152 \\
21481 & 00057 \\
21487 & 21321 \\
21491 & 44543 \\
21499 & 57364 \\
21503 & 40475 \\
21517 & 32365 \\
21523 & 77053 \\
21559 & 47601 \\
21563 & 07120 \\
21569 & 34402 \\
21577 & 66332 \\
21589 & 37570 \\
21599 & 75740 \\
21601 & 45246 \\
21613 & 75545 \\
21647 & 47635 \\
21673 & 52310
\end{tabular}

\begin{tabular}{cc}
$P$ & $R$ \\
\hline 21683 & 00532 \\
21701 & $P R 1 M E$ \\
21713 & 25422 \\
21737 & 70117 \\
21739 & 32026 \\
21751 & 64730 \\
21757 & 55670 \\
21773 & 22321 \\
21799 & 22140 \\
21851 & 55774 \\
21859 & 44313 \\
21881 & 67101 \\
21911 & 26047 \\
21991 & 03446 \\
22003 & 75446 \\
22013 & 46152 \\
22027 & 72245 \\
22037 & 31565 \\
22051 & 45260 \\
22067 & 56051 \\
22091 & 72501 \\
22111 & 37114 \\
22129 & 56330 \\
22133 & 34153 \\
22153 & 26761 \\
22189 & 43203 \\
22229 & 45706 \\
22283 & 12633 \\
22303 & 23264 \\
22307 & 45066 \\
22367 & 17214 \\
22391 & 02566 \\
22441 & 50616 \\
22469 & 03633 \\
22541 & 27254 \\
22571 & 00214 \\
22573 & 61446 \\
22613 & 62600 \\
22621 & 31456 \\
22637 & 16617 \\
22639 & 64640 \\
22643 & 47455 \\
&
\end{tabular}

\begin{tabular}{cc}
$P$ & $R$ \\
\hline 22651 & 64546 \\
22669 & 04145 \\
22691 & 16706 \\
22697 & 23007 \\
22699 & 47767 \\
22721 & 77111 \\
22741 & 36173 \\
22783 & 36067 \\
22787 & 31203 \\
22811 & 21766 \\
22817 & 70745 \\
22859 & 26653 \\
22861 & 01711 \\
22871 & 36761 \\
22877 & 25526 \\
22921 & 77227 \\
22961 & 07411 \\
22973 & 56750 \\
23011 & 04607 \\
23039 & 20007 \\
23059 & 31241 \\
23071 & 37716 \\
23117 & 72614 \\
23159 & 12223 \\
23173 & 42766 \\
23189 & 77456 \\
23201 & 56151 \\
23203 & 21500 \\
23209 & $P R 1 M E$ \\
23227 & 05321 \\
23291 & 15151 \\
23357 & 53157 \\
23431 & 41570 \\
23447 & 25202 \\
23473 & 60476 \\
23509 & 32462 \\
23537 & 72274 \\
23549 & 63457 \\
23561 & 12032 \\
23563 & 37447 \\
23581 & 15772 \\
23623 & 45666 \\
&
\end{tabular}

\begin{tabular}{cc}
$P$ & $R$ \\
\hline-3627 & 30230 \\
23633 & 20166 \\
23669 & 22751 \\
23677 & 15473 \\
23687 & 20104 \\
23689 & 35070 \\
23719 & 77150 \\
23741 & 61240 \\
23743 & 47034 \\
23753 & 67770 \\
23767 & 75375 \\
23773 & 41627 \\
23789 & 70524 \\
23813 & 76565 \\
23827 & 25176 \\
23869 & 75051 \\
23893 & 13651 \\
23899 & 37121 \\
23909 & 61071 \\
23911 & 35013 \\
23929 & 43422 \\
23971 & 44402 \\
23981 & 46073 \\
24001 & 45737 \\
24071 & 53203 \\
24077 & 61762 \\
24083 & 47001 \\
24091 & 55517 \\
24113 & 61244 \\
24121 & 20440 \\
24133 & 46206 \\
24151 & 42421 \\
24181 & 42161 \\
24197 & 62523 \\
24223 & 06747 \\
24251 & 24431 \\
24281 & 56776 \\
24407 & 42761 \\
24421 & 54676 \\
24443 & 56170 \\
24469 & 71364 \\
24473 & 37661 \\
24481 & 76122 \\
&
\end{tabular}

Using a revised form of the program, the first-named author then tested $M_{p}$ for $21701<p<24500$. He was able to eliminate $157 M_{p}$ using Wagstaff's table. Of the 125 remaining $M_{p}$ only $M_{23209}$ was found to be prime [7]. The test was completed on February 9, 1979 at 4:06 after 8:39:37 of CPU time. Lehmer and McGrogan later confirmed the result [4], [5] .

We give above two tables. The first lists Wagstaff's smallest factor for the 188 $M_{p}$ which did not require Lucas-Lehmer tests. The second lists the final Lucas residue, in octal $\left(\bmod 2^{15}\right)$, for the 169 Lucas-Lehmer tests that were computed.

On April 9, 1979, Dr. Slowinski and H. Nelson found $M_{44497}$ to be prime by using a similar implementation of the Lucas-Lehmer test on a CRAY 1 [11]. 
The amount of computation needed to check the primality of $M_{p}$ is $O\left(p^{3}\right)$. Here, the major computational effort is in squaring the $u_{k}$ since division by $2^{n}-1$ is readily accomplished by shifting. It may be possible to implement a faster multiplication method. For example, Schoenhage and Strassen [2] have an algorithm, based on fast Fourier transforms, which may be promising.

We would like to thank Dr. Jurca, Dr. Lehmer and Dr. Simon for their help, and Dr. Wagstaff for the use of his tables. We would also like to thank California State University at Hayward for use of their computer facilities.

California State University at Hayward

25800 Carlos Bee Boulevard

Hayward, California 94542

1. DONALD B. GILLIES, "Three new Mersenne primes, and a statistical theory," Math. Comp., v. 18, 1964, pp. 93-97.

2. DONALD E. KNUTH, Art of Computer Programming, Vol. 2, Addison-Wesley, Reading, Mass., 1963, pp. 269-275.

3. D. H. LEHMER, “On Lucas's test for the primality of Mersenne's numbers," J. London Math. Soc., v. 10, 1935, pp. 162-165.

4. D. H. LEHMER, Private communication with, University of California at Berkeley, Department of Mathematics, Berkeley, Calif. 94720.

5. STEVE McGROGAN, Private communication with, 19450 Yuma Drive, Castro Valley, Calif. 94546.

6. LAURA A. NICKEL \& CURT L. NOLL, "The 25 th Mersenne prime," Dr. Dobb's Journal, v. 4, issue 6, p. 6 .

7. CURT L. NOLL, "Discovering the 26th Mersenne prime," Dr. Dobb's Journal, v. 4, issue 6, pp. 4-5.

8. BRYANT TUCKERMAN, "The 24th Mersenne prime," Proc. Nat. Acad. Sci. U.S.A., v. 68, 1971, pp. 2319-2320.

9. BRYANT TUCKERMAN, Private communication with, International Business Machines Corporation, Thomas J. Watson Research Center, P.O. Box 218 , Yorktown Heights, New York 10598.

10. S. WAGSTAFF, JR., Private communication with, University of Illinois, Urbana, Illinois 61801 .

11. DAVID SLOWINSKI, "Searching for the 27th Mersenne prime," J. Recreational Math., v. 11,1979 , pp. $258-261$. 